\title{
Centro-parietal N200: An event-related potential component specific to Chinese visual word recognition
}

\author{
ZHANG John $\mathrm{X}^{1,2^{*}}$, FANG Zhuo ${ }^{2}$, DU YingChun ${ }^{3}$, KONG LingYue ${ }^{4}$, \\ ZHANG Qin ${ }^{5} \&$ XING Qiang ${ }^{6}$ \\ ${ }^{1}$ Department of Psychology, the Chinese University of Hong Kong, Hong Kong, China; \\ ${ }^{2}$ Medical College of Shantou University, Shantou 515041, China; \\ ${ }^{3}$ Key Laboratory of Behavioral Science, Institute of Psychology, Chinese Academy of Sciences, Beijing 100101, China; \\ ${ }^{4}$ International College of Chinese Language Studies, Peking University, Beijing 100871, China; \\ ${ }^{5}$ Department of Psychology, Capital Normal University, Beijing 100048, China; \\ ${ }^{6}$ Department of Psychology, Guangzhou University, Guangzhou 510006, China
}

Received August 8, 2011; accepted December 6, 2011

\begin{abstract}
Almost all written languages currently used in the world are alphabetic or phonologically-based scripts (e.g., English) whose word recognition involves discrimination of one-dimensional linear combinations of several dozens of letters or phonological units, except written Chinese whose vocabulary consists of a large number of block characters, the analysis of which relies on the extraction of 2-dimensional form information at multiple levels including radical, character, and multiple-character word. Whether the dramatic contrast between the 2 types of orthography leads to distinctive mental processes is a fundamental question in psycholinguistic research unanswered in the past three decades. Using event-related potentials and visual lexical decision tasks, we asked native Chinese speakers to discriminate between real Chinese words and pseudo- or non-words and observed a widespread negative deflection with centro-parietal focus elicited $200 \mathrm{~ms}$ post-stimulus onset. This N200 response showed a clear and large amplitude enhancement upon word repetition and seems to be specific to Chinese as no similar effects had been reported in word recognition studies involving alphabetic scripts under similar experimental conditions. Further evidence showed that this N200 could not be attributed to non-linguistic sensori-perceptual processes, nor phonological or semantic processes, but likely reflects very early identification of the orthography of individual words involving extensive and higher-level visual analysis. Recently the first author proposed a meaning-spelling theory of written Chinese vocabulary proposing that the Chinese and alphabetic scripts are the only 2 possible logical types of mature human writing systems, and that the former is more thoroughly a visual language compared with the latter and thus shall emphasize more of visual processing. Reinforcing each other, the meaning-spelling theory and the discovery of the centro-parietal N200 reveal the uniqueness of Chinese both theoretically and empirically, and provide strong arguments for the intrinsic distinction between written Chinese and alphabetic scripts.
\end{abstract}

N200, Chinese character, word recognition, orthography, meaning-spelling theory, alphabetic script

Citation: Zhang J X, Fang Z, Du Y C, et al. Centro-parietal N200: An event-related potential component specific to Chinese visual word recognition. Chin Sci Bull, 2012, 57: 1516-1532, doi: 10.1007/s11434-011-4932-y

Word is a fundamental unit of human language that in turn is central to high level intellectual behaviors. The thorough understanding of the cognitive and neural mechanisms underlying word recognition may bring breakthroughs to research on the fundamental principles of information pro-

*Corresponding author (email: jxzhang@cuhk.edu.hk) cessing in human brain. Reading is a highly emphasized and much cultivated core cognitive skill in modern society. The thorough understanding of the cognitive and neural mechanisms underlying written word recognition shall bring far-reaching impact on reading education. Distinct from the thousands of alphabetic or phonologically-based writing systems currently in use around the world, written Chinese 
is the only direct descendent from an endogenous ancient script, using a set of non-alphabetic block characters the structure of which had matured thousands of years before [1]. Chinese thus provides a precious sample for language study and comparative research across Chinese and alphabetic scripts could help to reveal the universal properties of human language.

Whether some characteristics of written Chinese, for example, the 2-dimensional pattern-like structure of its basic unit characters, would lead to unique mental processes has long been considered. Some early empirical psychological research suggested that character processing may involve more of the right hemisphere, compared with alphabetic scripts [2,3]. Later work based on brain-lesioned patients led to similar proposals [4]. Since 1980s, researchers around the world including that from mainland China, Taiwan, Hong Kong, and other places have conducted extensive psychological research on Chinese processing, expanding from traditional purely behavioral paradigms to more advanced cognitive neuroscience techniques, such as eventrelated potentials (ERP) and functional magnetic resonance imaging (fMRI) [5-7]. The new techniques enable more direct recording of neural activities associated with cognitive processes and offers a possibility to overcome limits when indirectly inferring brain activity from behavioral indices [8]. Linguists are also starting to use cognitive neuroscience methods to examine traditional linguistic topics [9]. However, with about three decades of research, including recent work in cognitive neuroscience, no reliable and well-accepted neural markers have been identified to distinguish word recognition across Chinese and other alphabetic scripts such as English [10-14]. Occasional reports of cross-script differences in brain activation are either not well-replicated or uncertain as to whether they do reflect unique processing for Chinese word recognition.

Assuming that there are indeed unique processing mechanisms for reading Chinese words, there may be some reasons why we failed to find them. As its main subject, psycholinguistic research over the past half a century has been primarily concerned with the English language, and research on visual word recognition has also been focused on simple words in English [15]. Following this tradition, word recognition research in Chinese has been mainly on single-character words as well [16-18], which are, however, not the most characteristic of the Chinese vocabulary. According to a recent corpus study (www.chineseldc.org), one-character words amount to only $2.8 \%$ of the total Chinese vocabulary, while 2- and 3-character words amount to $63.9 \%$ and $17.5 \%$ respectively. Theoretically, multiplecharacter word is a core feature of Chinese and shall receive more attention. There were in fact relatively few studies on multiple-character words, mostly with behavioral paradigms [19], even fewer with neuroscience methods. In addition, language processing, in particular, word recognition, involves very rapid processes that are often completed within a few hundred milliseconds. The most attended fMRI techniques that currently dominate cognitive neuroscience research are associated with a temporal resolution in a range of seconds, lacking the power to discriminate different stages in the complex recognition process. With its superior temporal resolution at the order of millisecond, it is possible to reveal the time course of word recognition with the ERP technique [20,21]. However, due to its weakness in spatial resolution, ERP research on Chinese word recognition did not receive due attention and a systematic examination of the basic effects in word recognition is still lacking.

In the past few years, we have conducted a series of ERP studies on the recognition of 2-character Chinese words, leading to the observation of a new electrophysiological response [22,23] that may be a possible neural indicator of distinctive processing mechanisms across Chinese and alphabetic scripts. We will present 6 experiments next to describe the basic characteristics of this response and to identify its associated cognitive process. A typical visual lexical decision task was used in all experiments in which native Chinese speakers were asked to discriminate between 2character real Chinese words and pseudo- or non-words.

\section{Experiment 1: Early ERP responses during 2-character Chinese word recognition}

\subsection{Methods}

Sixteen college students ( 8 males/ 8 females, age range from 21 to 28 years, mean age 24.2 years) who were fluent native Chinese speakers participated in this study. All were righthanded with normal or corrected-to-normal vision and none reported any history of neurological or psychiatric disorders. For all experiments reported here, written informed consent was obtained from each participant in accordance with a research protocol approved by the IRB board of the Chinese University of Hong Kong.

Participants sat in a dimly lit and sound-attenuated room facing a computer screen about $0.7 \mathrm{~m}$ away, resting their left and right index fingers on the $\mathrm{F}$ and $\mathrm{J}$ keys of a keyboard. They were asked to fixate a central fixation and to relax and minimize unnecessary head motions and avoid blinking during stimulus presentation and response making. The 2-character stimuli included 192 test items, half real words and half pseudo-words. Matching the real words in physical stimulus properties, the pseudo-words were constructed by re-combining the real word characters preserving character position, such as “技究”. They were not homophonic to any real words. After practice, each participant completed 3 testing sessions, with brief rest between 2 sessions.

Each session contained 64 items, including 16 high frequency words (mean frequency 765 , mean stroke number 13.9), 16 low frequency words (mean frequency 21, mean stroke number 17.6), and 32 pseudo-words, all randomly 
intermixed. Word frequency information was taken from the Modern Chinese Word Frequency Dictionary [24] and the unit was occurrence per million. Each trial started with a fixation cross for $500 \mathrm{~ms}$, followed by the presentation of a word for $400 \mathrm{~ms}$, centered at the screen center, subtending a visual angle of $4.3^{\circ} \times 2.3^{\circ}$. Participants were to decide whether or not the stimulus was a real Chinese word and to press either a left or right key to indicate their response within a 1900-ms response window from stimulus onset. Both response speed and accuracy were emphasized. The response and key mappings were counterbalanced acrosssubjects. Between the response and the fixation onset for the next trial, there was a blank interval varying randomly from 600 to $800 \mathrm{~ms}$.

EEGs were recorded from the scalp with 64 nonpolarizable $\mathrm{Ag} / \mathrm{AgCl}$ sintered electrodes in preconfigured caps, using the Neuroscan EEG system with a sampling rate of $500 \mathrm{~Hz}$ and band-pass filtering (range $=0.1-70 \mathrm{~Hz}$ ). The electrode sites followed the extended 10-20 convention. Both horizontal and vertical EOGs were recorded with the former placed on temporal sides of the 2 eyes and the latter placed below the left eye and above the left eye brow. The impedance in all electrodes was less than $5-\mathrm{k}$ ohm. The recordings were physically referenced to the nose tip and re-referenced offline to the mean of the 2 mastoids. Each averaging epoch lasted $1000 \mathrm{~ms}$ including a 100 -ms interval prior to stimulus onset for baseline correction. Average ERPs were computed offline for correct trials free of ocular and movement artifacts using standard procedures in the Scan 4.3 software (http://www.neuroscan.com). Band-pass filtering was applied with cut-off frequencies of 0.1 and $30 \mathrm{~Hz}$. For ocular artifacts, the parameters were set at 20 for minimum number of sweeps, 400 for blink duration, and $10 \%$ for thresholding. The amplitude rejection criterion was set at $\pm 100 \mu \mathrm{V}$. The mean number of trials rejected due to eye movement or artifacts was less than $10 \%$, also true for all following experiments.

\subsection{Results and discussion}

Across all participants, the mean response time and error rates in the high frequency real word condition were significantly faster and lower than the low frequency real word condition (548 vs. $621 \mathrm{~ms}, 1.4 \%$ vs. $6.3 \%, P<0.01$ ), both significantly faster than the pseudo-word condition $(718 \mathrm{~ms}$, $11.1 \%, P<0.01)$. The incorrect trials were excluded from the response time computation. These behavioral results replicated the classical lexical effect and the word frequency effect, confirming the validity of the experimental control. Figure 1 shows in some representative electrodes the averaged ERP responses using different colors for the 3 conditions. For the negative wave peaking in between 300 and $400 \mathrm{~ms}$, we selected 4 rows and 3 columns of 12 electrodes (FC1, FCz, FC2; C1, Cz, C2; CP1, CPz, CP2; P1, Pz, P2) and computed the average amplitude in this $100-\mathrm{ms}$ time window. As the mean amplitude was significantly lower in the pseudo-word condition than the real word condition, in the low-frequency condition than the high frequency condition, this deflection was identified as the widely-studied N400 component associated with semantic processing [25]. As can be seen from Figure 1, in most electrodes depicted, there were the N1, P2 and N400 responses often reported in the word recognition literature [15,26,27]. As these 3 components were not the focus of the present study and their results pattern conformed to literature findings, their descriptions would be brief and not detailed below.

New to our study was a negative deflection peaking at $216 \mathrm{~ms}$, called N200 for easy reference. The peak amplitude of this N200 was not very large, but it showed a wide scalp distribution, present in frontal, central, and parietal regions. A $40 \mathrm{~ms}$ time window was taken centered at its peak time point extending $20 \mathrm{~ms}$ bilaterally. Pooling over the 12 electrodes described above, the averaged amplitude within this time window was no different across the high, low frequency real word, and the pseudo-word conditions ( $P$ values greater than 0.5 for all pairwise comparisons).

There has been only limited ERP work on 2-character visual words in Chinese. After noting the N200 response, we were able to find some previous studies documenting similar responses. They were however unattended by the researchers, possibly because of its small magnitude, possibly because that researchers were mainly concerned with later semantic processing (e.g., concreteness, noun vs. verb) and the associated N400, as opposed to early lexical processing [26,28]. On the other hand, studies using single-character words typically did not show this N200 response [27,29]. Future studies with enhanced power are needed to find out whether single-character words do not elicit N200 at all, or they elicit a much weaker response compared with 2-character words.

\section{Experiment 2: Comparison of ERP responses between Chinese and Korean 2-character words in the $\mathrm{N200}$ time window}

\subsection{Design}

One possibility was that the N200 observed in Experiment 1 may not be language-related but originated from some physical properties of the 2-character words, such as they involved the juxtaposition of 2 visual stimuli. To test this sensori-perceptual explanation of N200, here we asked Chinese participants to distinguish between 2-character Chinese words and 2-character Korean words that were randomly intermixed. Influenced by the Chinese script, Hangeul characters consist of strokes and radicals packed into a square shape (Figure 2). In terms of both its basic units and overall configuration, Korean words closely match the Chinese words, although they are nonsense visual symbols (or non-words) to our Korean-naïve participants. This 
experiment was intended to see whether this kind of stimuli with similar perceptual properties as the Chinese words would elicit N200.

\subsection{Methods}

A new group of 16 college students ( 8 males/ 8 females, age range 19-24 years, mean age 22.2 years) meeting the same criteria as Experiment 1 participated in this experiment. None reported any experience of ever learning written or spoken Korean. The procedure, experimental parameters, and recordings and analysis of the EEG signals were the same as in Experiment 1 except what was described below. The EEG recording was conducted with a 64 channel BrainAmp amplifier from the Brain Products Company (USA). Following the system's default setup, FCz was used as the physical reference during recording. The EEG signals were re-referenced offline using the averaged signal from the 2 mastoids and the waveform in $\mathrm{FCz}$ was computed using interpolation.

Each participant completed 2 testing blocks, each including 72 word stimuli, half being Chinese and half being Korean, randomly intermixed. The mean word frequency for the Chinese words was 37.9 and the stroke number was matched across the Chinese and the Korean words (11.0 vs. $11.2, P>0.5)$. In each trial, a word was presented centrally and participants were required to decide as quickly and as accurately as possible whether it was a Chinese word or not and indicate their response by pressing one of 2 buttons.

\subsection{Results and discussion}

Data from one participant were discarded due to technical breakdown. For the remaining 15 participants, the mean response time and error rates were comparable for the Chinese and Korean words (504 vs. $514 \mathrm{~ms}, P>0.2$; 3.6\% vs. $3.8 \%, P=0.5)$. Figure 2 shows the averaged ERP waveforms in some representative electrodes for both the Chinese and Korean conditions. As in Experiment 1, most electrodes showed N1, P2 and N400 responses. The first 2 components are known to reflect basic sensori-perceptual processing non-specific to language [30]. The results that their amplitudes and latencies were highly comparable across the 2 stimulus conditions, particularly in the occipital regions, indicate that the 2 types of stimuli were matched in their physical properties, such as visual complexity.

The N400 amplitude for the Korean condition was significantly larger than the Chinese condition, in line with a general characteristic of N400 to be more salient for nonsense materials. The Chinese words elicited at $218 \mathrm{~ms}$ a clear and widespread N200, replicating the basic finding in Experiment 1. In comparison, no similar responses were found for the Korean words the waveform of which changed monotonically and smoothly in between the 2 peaks of P2 and N400. The results argue against the sensori-perceptual interpretation of $\mathrm{N} 2$ and suggest that the juxtaposition of 2 visual items is not sufficient to elicit N200. N200 could not be attributed to some special, non-linguistic property of the 2-character Chinese words, because it could not be elicited by the Korean words with similar physical properties. In other words, that the Chinese words but not the Korean words elicited N200 should be because the former constituted meaningful language materials to the participants while the second did not. Therefore, N200 should reflect some process related to linguistic processing.

\section{Experiment 3: Effect of repetition priming on N200}

\subsection{Design}

The following 4 experiments adopted a neural priming paradigm to investigate the specific linguistic processing underlying the N200 response. This type of paradigm combines the traditional priming method and cognitive neuroscience techniques to examine how a stimulus would affect neural responses to a related stimulus presented subsequently, and has been widely-recognized to be a powerful tool in revealing the brain mechanisms for object and word recognition [31]. The type of priming used in the present experiment was repetition priming where the first presented prime stimulus was identical to the later presented target stimulus. Typically, such a situation could lead to the largest priming effect [32].

As in Experiment 2, we would present a series of items intermixing Chinese and Korean 2-character words and asked participants to discriminate between the two. Differently, in the stimulus sequence, there would be 4 types of prime-target pairs. Among 2 neighboring stimuli (the $n$ and $n+1$ stimulus), the first would be the prime stimulus and the second the target stimulus. Depending on the relationship between the prime and the target, we defined the following conditions: (1) real control condition, the two were unrelated real words; (2) real repeat condition, the two were the same real word; (3) Korean control condition, the two were unrelated Korean words; (4) Korean repeat condition, the two were the same Korean word. Based on results from Experiment 2, we expected that the Chinese words would elicit N200 but the Korean words would not. The focus was to examine whether repetition priming would affect N200 or the ERP responses in the N200 time window.

\subsection{Methods}

A new group of 20 college students (9 males/11 females, age range 21-26 years, mean age 23.7 years) meeting the same criteria as in Experiment 3 participated in this experiment. The procedure, experimental parameters, and recordings and analysis of the EEG signals were the same as in Experiment 3 except what was described below. 

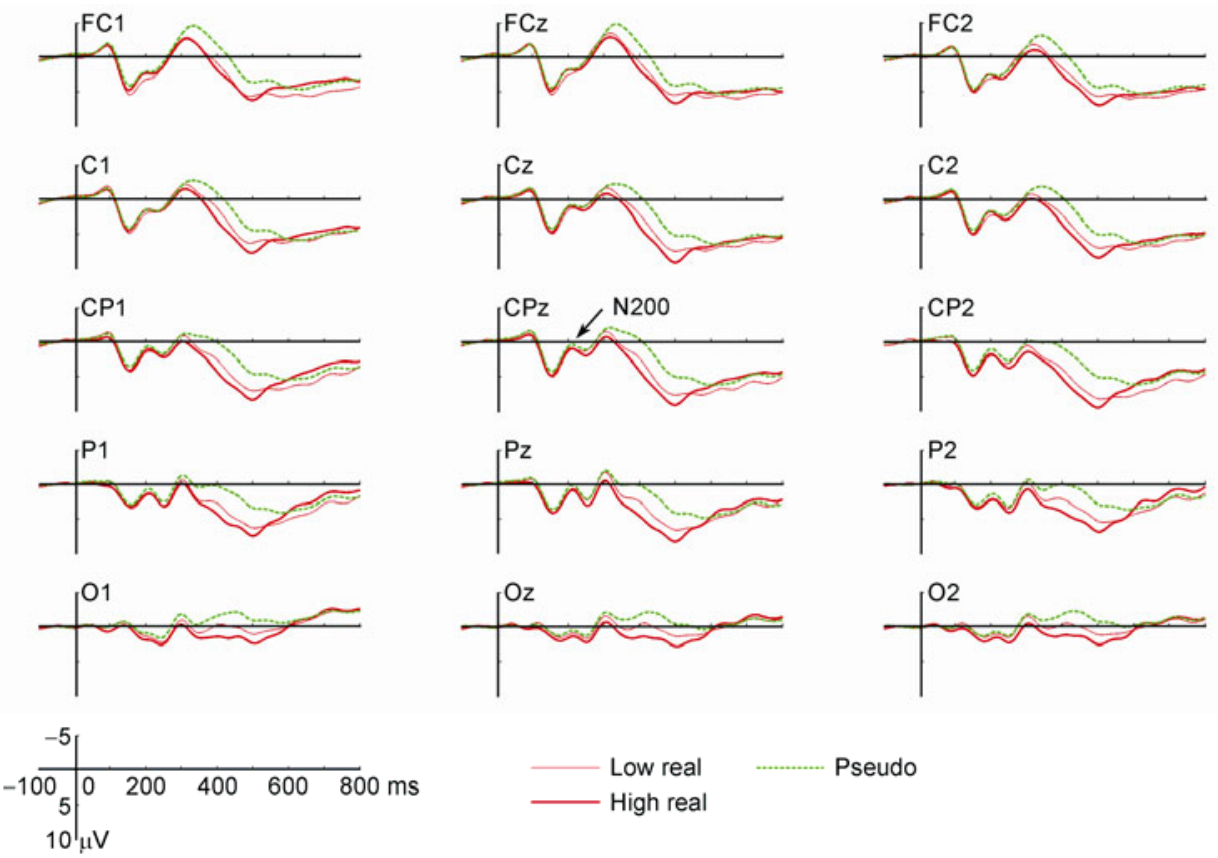

Figure 1 Averaged ERP waveforms for all conditions in Experiment 1.
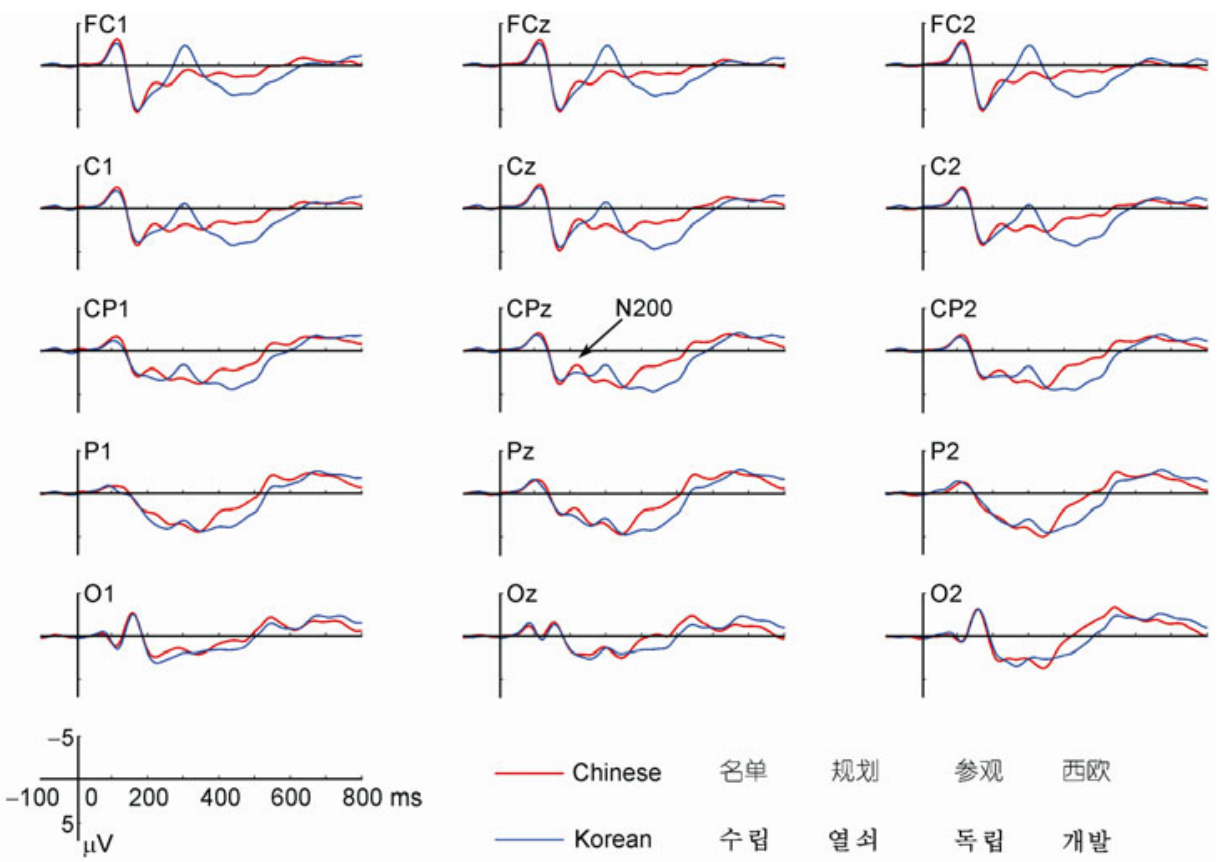

Figure 2 Averaged ERP waveforms for all conditions in Experiment 2.

The test stimuli included 90 2-character Chinese words (mean frequency 50.2) and 90 2-character Korean words, matched in stroke number (12.0 vs. 11.7). Sixty words were randomly selected from the Chinese words for the repeat condition. The words were divided into 3 groups, each used to construct 20 pairs of repeat word pairs for a repetition lag of 0,1 and 2 (the same word was presented 2 times with 0 , 1 , or 2 intervening items). The same procedure was applied to the Korean words to construct 3 groups of repeat word pairs. These repeat word pairs would be randomly intermixed with the remaining 30 Chinese words and 30 Korean words making a sequence of 300 items $(60 \times 2+30+60 \times 2+$ $30)$. This sequence was then divided into 4 test blocks of 75 stimuli. For more data points, the same process was repeated 2 more times so that each participant completed 12 blocks so constructed with a brief rest between 2 blocks. 
Repetition lag was a factor considered in the initial design, although the final results did not reveal any meaningful effects at lag 1 and 2 . The following discussion would be confined to the lag 0 pairs only, or pairs of immediate repetition. For all 4 experimental conditions, the behavioral and ERP analysis were focused on the target stimuli. There were a total of 60 data points for both the Chinese and the Korean repeat conditions. For their respective control condition, the total number of data points was about 110 . Due to the nature of the stimulus construction process, there were more neighboring pairs where the 2 words were unrelated than where the two were the same.

\subsection{Results and discussion}

The behavioral results showed strong priming effects where mean response time and error rates were significantly faster and lower for the target word in the Chinese repeat condition, compared with the Chinese control condition (482 vs. $506 \mathrm{~ms}, P<0.001 ; 2.6 \%$ vs. $6.5 \%, P<0.001)$. No priming effect was present for the Korean words (513 vs. $518 \mathrm{~ms}$, $P>0.1 ; 5.0 \%$ vs. $4.2 \%, P>0.3)$.

Figure 3 shows the averaged ERP waveforms in some representative electrodes for all 4 conditions. As in Experiment 2, for the control conditions, most electrodes showed $\mathrm{N} 1, \mathrm{P} 2$ and N400 responses. The first 2 responses were highly comparable across the Chinese and Korean conditions, indicating a match on physical stimulus properties. Following the same analysis procedure as in Experiment 1, within the $100-\mathrm{ms}$ time window from 300 to $400 \mathrm{~ms}$, the N400 amplitude for the Korean condition was significantly larger than the Chinese condition, in line with a general characteristic of N400 to be more salient for nonsense materials. For the Chinese words, N400 showed a significant amplitude reduction in the repeat condition than in the control condition, demonstrating the well-established repetition priming effect in the word recognition literature [32], indicating that a word's first presentation facilitated greatly its semantic processing at the second presentation. For the Korean words, there was no repetition effect in N400, presumably because there were no semantic representations for these words to the Chinese participants and consequently no facilitated semantic processing at repetition.

For the control condition, the Chinese words elicited a clear N200 response but the Korean words did not. This replicated the basic findings in the first 2 experiments. Critically, compared with the control condition, the Chinese words elicited a significantly enhanced N200 (more negative) under the repeat condition ( 0.6 vs. $1.3 \mu \mathrm{V}, P<0.005)$. The scalp distribution of this repetition effect was widespread with a focus in central and parietal regions. In comparison, in the same time window of this effect, the control and repeat conditions for the Korean words were no different at all (1.7 vs. $1.9 \mu \mathrm{V}, P>0.1)$. The choice of time window and electrodes in these analysis was the same as in
Experiment 1.

$\mathrm{Xu}$ [33] presented a series of words to Chinese participants with English as their second language and asked them to make semantic judgments on these words. One of their conditions involved immediate repetition of 2-character Chinese words and indeed in that condition they recorded a N200 response at the word's first presentation and an enhanced N200 at the word's repetition. Their results were an independent replication of the present findings. However, $\mathrm{Xu}$ was mainly concerned with the effect of bilingual processing on N400 and attributed the N200 she observed to a non-linguistic inhibition process, similar to the inhibitory $\mathrm{N} 2$ often studied with the Go/No-Go paradigm. In fact, the $\mathrm{N} 200$ s documented in her study and the present one were considerably earlier than the inhibitory $\mathrm{N} 2$ (by about $100 \mathrm{~ms}$ ). Another reason the inhibitory $\mathrm{N} 2$ could not explain these $\mathrm{N} 200$ s was that the tasks $\mathrm{Xu}$ and we used were standard language tasks not involving inhibitory processes.

\section{Experiment 4: Phonological priming and N200 repetition enhancement}

\subsection{Design}

In Experiment 3, when 2-character Chinese words were repeated, other than the well-known N400 amplitude reduction effect, there was a new effect of N200 enhancement. The processing of words as linguistic materials involves 3 dimensions of orthography, phonology, and semantics. The repetition effect of N200 may originate from processing along one or more of the 3 dimensions. In the present experiment, we examined whether phonology contributes to the effect by manipulating the relationship between the prime and the target to make them identical in phonology (or being homophones to each other) but unrelated in orthography and semantics.

As in Experiment 3, the task was lexical decision except that the Korean words were replaced with pseudo-words constructed with 2 real characters as described in Experiment 1 . Depending on the relationship between 2 neighboring stimuli, the stimulus sequence contained the following 4 types of prime-target pairs: (1) real control condition, the two were unrelated real words; (2) real repeat condition, the two were the same real word; (3) phonological priming condition, the two were homophones (same tone as well) but different in orthography and unrelated in semantic meaning; (4) pseudo-word control condition, the prime was a real word and the target was an unrelated pseudo-word. The first two conditions were the same as in Experiment 3 while the latter two were newly added.

\subsection{Methods}

A new group of 20 college students (11 males/9 females, 
age range 21-25 years, mean age 23.0 years) meeting the same criteria as in Experiment 1 participated in this experiment. The procedure, experimental parameters, and recordings and analysis of the EEG signals were the same as in Experiment 3 except what was described below.

The test stimuli included 60 pairs of 2-character Chinese homophonic words (mean frequency 6.2, 6.1, mean stroke number $16.7,16.6), 60$ real words to use for the repeat condition (mean frequency 6.2, mean stroke number 16.6), 400 real words as control items (mean frequency 5.9, mean stroke number 16.7), and 120 2-character pseudo-words (mean stroke number 16.8). Stroke number was matched across all groups and word frequency was matched across all real word groups. Each participant completed 10 test blocks, each of 76 trials, including 6 homophonic pairs, 6 repeat pairs, and 40 control real words, and 12 pseudowords, randomly intermixed. For all 4 experimental conditions, the behavioral and ERP analysis were focused on the target stimuli. There were a total of 60 data points for both the repeat condition and the phonological priming condition. For the first condition, i.e., the real control condition, the corresponding number was 410. As in Experiment 3, due to the nature of the stimulus construction process, there were more neighboring pairs where the 2 words were unrelated than where the two were the same.

As our main focus was on the real words, different from Experiment 3, here participants were asked to make a keypress response only to the pseudo-words but withhold response to the real words. The pseudo-words were in fact the non-essential filler materials with much fewer number of occurrences than the real words. This way, more trials could be allocated to the real word conditions for more data points, although the problem was that no response time could be collected for the real words. As a makeup, a new group of 16 participants from the same subject population were recruited for a separate behavioral experiment that was identical to the ERP one except that they were to press one key for the real words and another key for the pseudo-words. The results showed highly significant repetition priming effect, the real repeat condition was faster in response time and lower in error rate than the real control condition (523 vs. $633 \mathrm{~ms}, P<3 \times 10^{-9} ; 2.1 \%$ vs. $\left.3.6 \%, P<0.05\right)$. The phonological priming effect was not significant $(629 \mathrm{vs} .633 \mathrm{~ms}$, $P>0.5 ; 4.8 \%$ vs. $3.6 \%, P>0.2)$. The real control condition was faster and more accurate than the pseudo-word condition (633 vs. $861 \mathrm{~ms}, P<4 \times 10^{-8} ; 3.6 \%$ vs. $19.0 \%, P<5 \times 10^{-5}$ ).

\subsection{Results and discussion}

For the behavioral results, the mean response time was $980 \mathrm{~ms}$ for the pseudo-words with a mean error rate of $8.8 \%$. The mean false alarm rate for the real words was $3.2 \%$. As shown in Figure 4, the target words in the real control and the pseudo-word conditions elicited N1, P2, N200, and N400 with a similar pattern as in Experiments 2 and 3, which would not be described in detail. Further, there was no difference in N200 amplitude between the real control and the pseudo-word conditions, replicating what was found in Experiment 1 that N200 amplitude was unaffected by the lexicality of the whole word. Following the same analysis procedure as in Experiment 1, we found a significant N400 amplitude reduction and a significant N200 amplitude enhancement $(1.5$ vs. $2.5 \mu \mathrm{V}, P<0.0005)$ in the real repeat condition, compared with the real control condition. This replicated the basic finding in Experiment 3. The N200 repetition effect was again very salient in central and parietal regions but tended to more salient in the frontal region when compared with that in Experiment 3.

Phonological priming also led to some significant N400 amplitude reduction, consistent with similar findings previously reported with single-character words [27], indicating that the present phonological manipulation was effective. As the most critical result, phonological priming, however, did not have any effect on N200, no statistical difference was found in N200 amplitude between the phonological priming condition and the real control condition (2.6 vs. $2.5 \mu \mathrm{V}, P>0.5)$.

\section{Experiment 5: Semantic priming and N200 repetition enhancement}

\subsection{Design}

Similar to Experiment 4, here we further examined whether semantic processing affects the N200 repetition effect by manipulating the relationship between the prime and the target to make them related in semantic meaning but not in orthography and phonology. As in Experiment 4, the task was lexical decision presenting participants with a series of 2-character real and pseudo-words for discrimination. The stimulus sequence contained the following 5 types of primetarget pairs: (1) real control condition, the two were unrelated real words; (2) real repeat condition, the two were the same real word; (3) semantic priming condition, the two were semantically related but different in orthography and phonology; (4) pseudo-word control condition, the two were unrelated pseudo-words; (5) pseudo-word repeat condition, the two were the same pseudo-word. The first two conditions were the same as in Experiments 3 and 4 while the latter three were newly added.

\subsection{Methods}

A new group of 20 college students (15 males $/ 5$ females, age range 19-25 years, mean age 22.3 years) meeting the same criteria as in Experiment 1 participated in this experiment. The procedure, experimental parameters, and recordings and analysis of the EEG signals were the same as in Experiment 3 except what was described below.

The test stimuli included 50 pairs of 2-character Chinese 

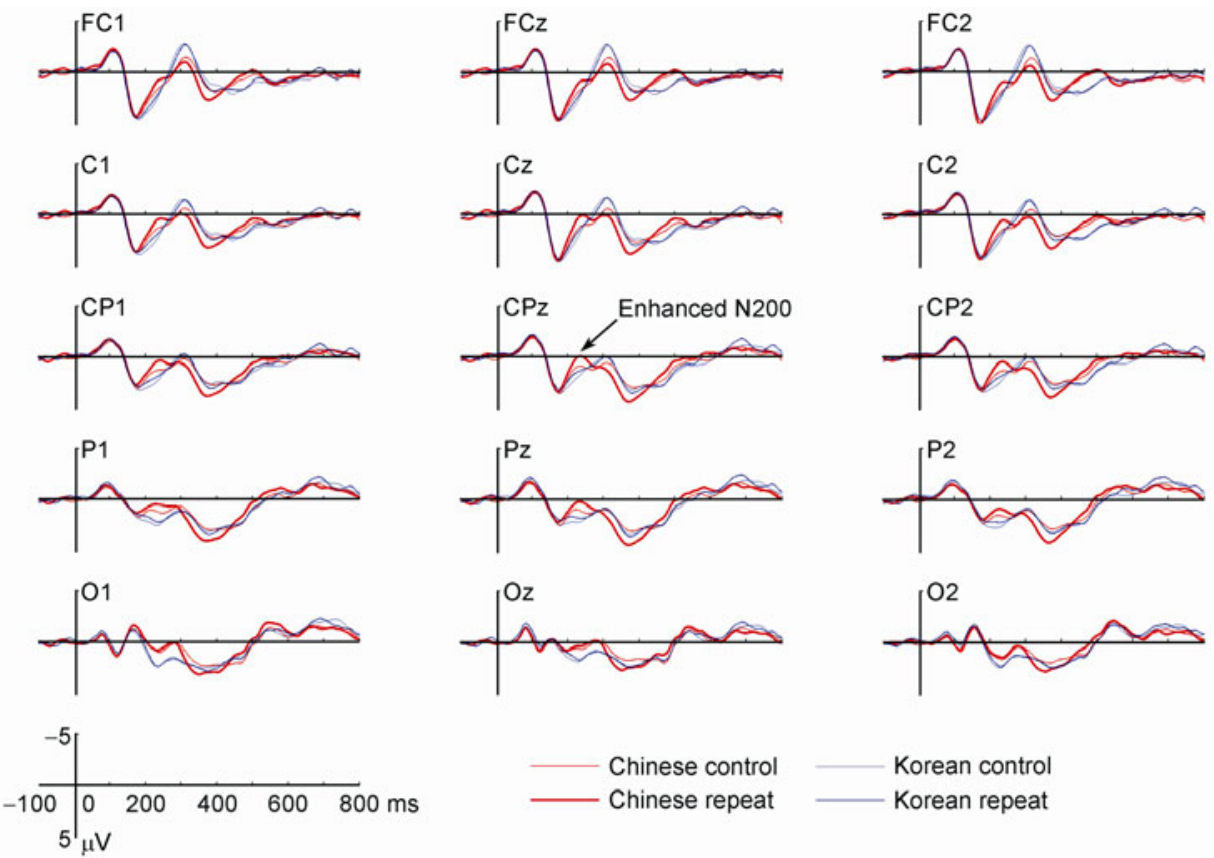

Korean control Korean repeat

Figure 3 Averaged ERP waveforms for all conditions in Experiment 3.
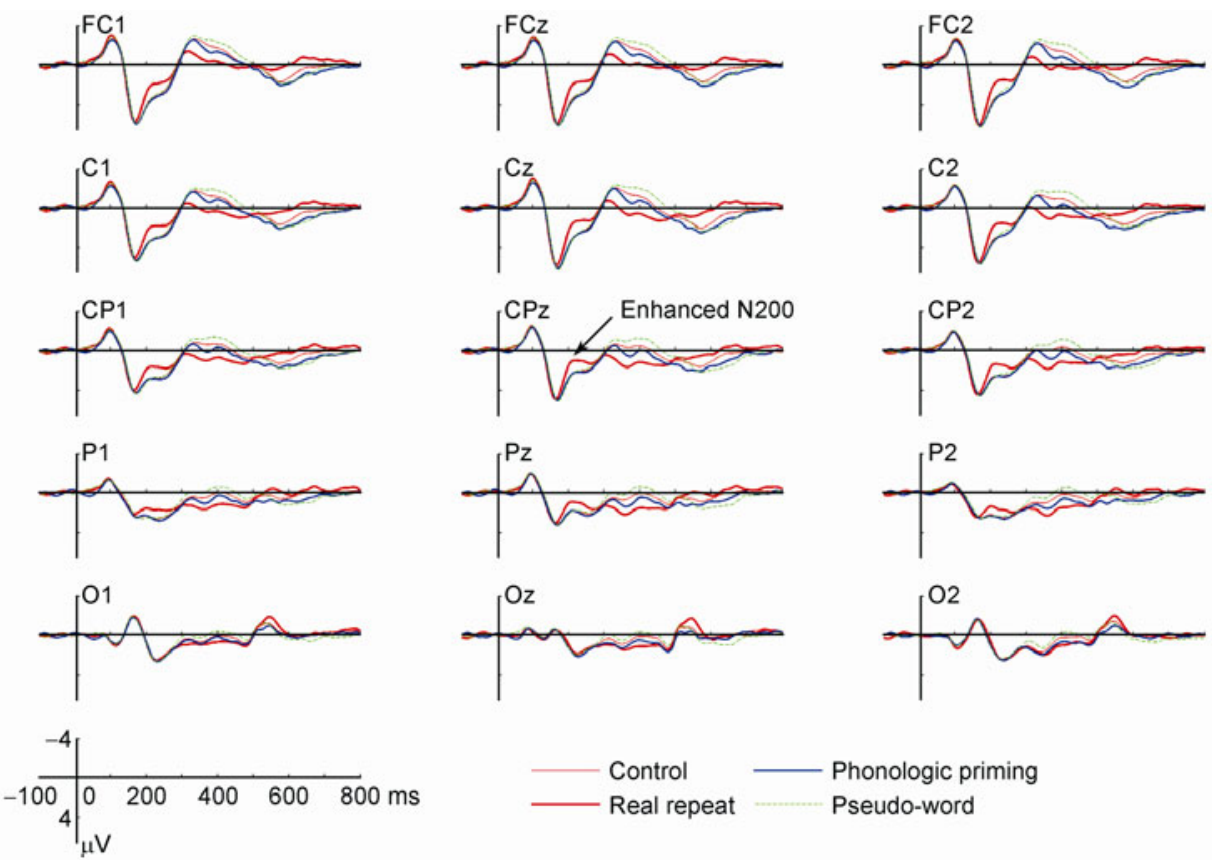

Figure 4 Averaged ERP waveforms for all conditions in Experiment 4.

words that were semantically related (mean frequency 34.2 , 34.3 , mean stroke number $17.1,16.8)$, 50 real words to use for the real repeat condition (mean frequency 32.9, mean stroke number 16.7), 50 pseudo-words to use for the pseudo-word repeat condition (mean stroke number 17.2), 200 real words as control items (mean frequency 31.7, mean stroke number 16.7), and 300 pseudo-words (mean stroke number 16.8). All items consisted of 2 characters. Stroke number was matched across all groups and word frequency was matched across all real word groups. As rated by 16 participants from the same subject population using a 5 -point scale ( 1 for unrelated in meaning and 5 for highly related), the mean semantic relatedness between the words pairs in the semantic priming condition was $3.84(\mathrm{SD}=0.27)$.

Each participant completed 10 test blocks, each of 80 trials, including 5 semantically related pairs, 5 real word 
repeat pairs, 5 pseudo-word repeat pairs, and 20 real and 30 pseudo-words as control items, randomly intermixed. Half of the trials were with a real word and the other half with a pseudo-word. For all 5 experimental conditions, the behavioral and ERP analysis were focused on the target stimuli. There were a total of 50 data points for the 2 repeat conditions and the semantic priming condition. For the real control and pseudo-word control conditions, the corresponding numbers were 100 and 150 . The reason was the same as in Experiments 3 and 4 that, due to the nature of the stimulus construction process, there were more neighboring pairs where the 2 words were unrelated than where the two were the same.

\subsection{Results and discussion}

The behavioral results showed clear effects of repetition priming and semantic priming. Mean response time and error rates were significantly faster and lower for the real repeat condition than the real control condition (589 vs. 722 ms, $P<5 \times 10^{-12} ; 2.1 \%$ vs. $\left.7.8 \%, P<1 \times 10^{-6}\right)$. The same pattern was found for the semantic priming condition (661 vs. $722 \mathrm{~ms}, P<3 \times 10^{-9} ; 1.8 \%$ vs. $7.8 \%, P<5 \times 10^{-6}$ ). The mean response time for the pseudo-word repeat condition was also significantly faster than the pseudo-word control condition (650 vs. $788 \mathrm{~ms}, P<1 \times 10^{-11}$ ), while the mean error rates were comparable $(3.2 \%$ vs. $4.4 \%, P>0.1)$.

As shown in Figure 5, the target words in the real and the pseudo-word control conditions elicited N1, P2, N200, and N400 with a similar pattern as in Experiments 3 and 4, which would not be described in detail. Further, there was no difference in N200 amplitude between these 2 conditions, replicating what was found in Experiments 1 and 4 that N200 amplitude was unaffected by the lexicality of the whole word. Compared with the real control condition, there were a significant N400 amplitude reduction and a significant N200 amplitude enhancement in the real repeat condition ( 1.8 vs. $2.6 \mu \mathrm{V}, P<0.005)$, which replicated the basic findings in Experiments 3 and 4. The scalp distribution of this N200 repetition effect was close to that in Experiment 4.

Compared with the pseudo-word control condition, there was a significant reduction in N400 amplitude in the pseudoword repeat condition, although the effect size was smaller compared with the N400 repetition priming effect for the real words. Surprisingly, pseudo-word repetition also led to N200 enhancement (1.4 vs. $\left.2.5 \mu \mathrm{V}, P<5 \times 10^{-5}\right)$ with an effect size no different from the enhancement effect found for the real word repeat condition $(2 \times 2$ interaction $F(1,19)=1.0$, $P>0.3$ ). Compared with the real control condition (akin to a condition without semantic priming), the semantic priming condition showed a significant N400 amplitude reduction, replicating the classic N400 semantic priming effect [25]. As the most critical result, semantic priming, however, did not have any effect on N200, no statistical difference was found in N200 amplitude between the semantic priming condition and the real control condition ( 2.3 vs. $2.6 \mu \mathrm{V}, P>$ 0.3 ). As the effect of phonology and semantics were discounted in Experiments 4 and 5, it was suggested that the N200 enhancement effect should be associated with orthographic processing.

\section{Experiment 6: Partial orthographic repetition and $\mathbf{N 2 0 0}$ repetition enhancement}

\subsection{Design}

Through logical exclusion, results from Experiments 3 to 5 were used to eliminate the effect of phonology and semantics and to argue that N200 repetition enhancement reflects orthographic processing. To give a more direct test of this conclusion, here we manipulated the orthographic similarity between the prime and the target to see whether this similarity would affect the N200 repetition effect. As in Experiment 4, the task was lexical decision where participants were presented with a series of 2-character real words and pseudo-words and asked to discriminate between the two. As our main interest was about the real words, there were only very few pseud-words (consisting of 2 real characters) serving as the targets for detection.

The stimulus sequence contained 4 types of prime-target real word pairs: (1) control condition, the two were unrelated real words, such as “钱币-微弱” (money-weak); (2) repeat both condition, the two were the same real word, such as “思索-思索” (think-think); (3) repeat first condition, the two words shared the first character but not the second, such as “荣幸-荣华” (honored-prosperity); (4) repeat second condition, the two words shared the second character but not the first, such as “流利-互利” (fluent, mutually-benefiting). The first 2 conditions were the same as in Experiments 3, 4, and 5 . In the latter 2 newly added conditions, the prime and the target differed from each other at the whole word level. However, since they had one character in common, they constituted a pair with partial orthographic repetition because orthography of the single character was part of the orthography of the 2-character whole word.

\subsection{Methods}

A new group of 26 college students ( 4 males/22 females, age range $17-25$ years, mean age 20.2 years) meeting the same criteria as in Experiment 1 participated in this experiment. The procedure, experimental parameters, and recordings and analysis of the EEG signals were the same as in Experiment 4 except what was described below. Each participant completed 10 test blocks, each of 80 trials, including 5 repeat both pairs, 5 repeat first pairs, 5 repeat second pairs, 20 control pairs, randomly intermixed with 10 pseudo-words.

The test stimuli included 200 control pairs (prime: mean word frequency 7.2, first, second character mean stroke number $8.2,8.4$, mean character frequency 689 , 789; corre- 
sponding values for target: $7.2,8.5,8.6,916,572), 50$ repeat both pairs (prime: $6.8,8.7,8.9,466,523$; target: same as for prime), 50 repeat first pairs (prime: $6.8,8.6,8.1,619,638$; target: 7.0, 8.6, 7.9, 619, 738), 50 repeat second pairs (prime: 6.9, 9.1, 8.7, 418, 504; target: 7.4, 8.6, 8.7, 664, 504), and 100 pseudo-words (first, second character mean stroke number 8.6,8.2, mean character frequency 536,852). Both word and character frequency were in unit of occurrence per million. Stroke number was matched across all groups and word frequency was matched across all real word groups. For the control condition, the first character frequency of the prime word was significantly higher than other conditions. As there were many data points in this condition (200 trials), a test was conducted post-hoc sub-dividing these trials into 2 groups of high or low values in this frequency measure. The test failed to reveal any significant effect of this character frequency on the results described below.

As in Experiment 4, participants were asked to make a keypress response only to the pseudo-words but withhold response to the real words. There were very few pseudo-words serving as the detection target and this allowed more trials to be allocated to the real word conditions for more data points. For all 4 real word conditions, the behavioral and ERP analysis were focused on the target stimuli.

\subsection{Results and discussion}

For the behavioral results, the false alarm rates (as the correct response was not to respond) for all real word conditions were below 3\%, and there were no statistical differences across conditions. For the pseudo-words, the mean accuracy rate was $97.1 \%$, and the mean response time was $745 \mathrm{~ms}$. As shown in Figure 6, the target words in the real conditions elicited N1, P2, N200, and N400 with a similar pattern as the real word conditions in Experiments 3-5. Following the analysis procedure as described in Experiment 4, one-way ANOVAs (4 levels) were conducted on N200 and N400 amplitude, revealing significant effects (N200: $F(3,75)=11.3, P<0.001$; N400: $F(3,75)=18.2, P<$ $0.001)$. From the control, repeat second, repeat first, to repeat both conditions, N200 amplitude showed monotonic increase (more negative), being 4.2, 3.9, 3.4, and $2.8 \mu \mathrm{V}$ respectively, while N400 amplitude showed an opposite pattern, being $-0.8,0.1,0.4$, and $1.4 \mu \mathrm{V}$ for the 4 conditions respectively. Compared with the control condition, the repeat both condition showed a significant reduction in N400 amplitude $\left(t(25)=7.1, P<5 \times 10^{-7}\right)$, and at the same time a significant N200 enhancement $\left(t(25)=-6.1, P<5 \times 10^{-6}\right)$, duplicating results in Experiments 3, 4, and 5.

Compared with the control condition, the repeat first condition showed a significant $\mathrm{N} 400$ reduction $(t(25)=4.0$, $\left.P<5 \times 10^{-4}\right)$ as well as a N200 enhancement $(t(25)=-2.80$, $P<0.01)$; the repeat second condition showed a significant N400 reduction $(t(25)=3.4, P<0.005)$ and a non-significant N200 enhancement $(t(25)=-1.1, P=0.3)$. This indicates that for the repeat both condition when the prime and target words were of maximal orthographic similarity, the N200 enhancement effect was also larger, and that for the repeat first and repeat second conditions when there was partial repetition or smaller orthographic similarity between the prime and the target, the N200 enhancement effect was also smaller. This finding provides more direct evidence for the notion that N200 reflects orthographic processing. In either the repeat first or the repeat second conditions, the prime and the target were different words at the whole word level, the N200 enhancement for these partial repetition conditions therefore indicates that N200 may not only reflect whole word or lexical processing, but is more likely to be also associated with sub-lexical processing. The result that N200 enhancement was significantly smaller in the repeat first condition than in the repeat both condition $(0.6 \mathrm{vs} .1 .4 \mu \mathrm{V}$, $t(25)=-2.10, P<0.05)$ suggests that the repetition effect of the whole word cannot be fully accounted for by the repetition effect from the first character.

The N200 enhancement for the repeat first condition was larger than for the repeat second condition with boarder-line significance $(t(25)=-1.9, P=0.06)$. This indicates that N200 repetition of a 2-character word cannot be reduced to the repetition of its constituent characters, otherwise, one should expect similar repetition effects for the first and the second character. That repetition of the first character influenced N200 more than did the second character suggests that the first character was more important for the whole word than the second, or processing of the 2 characters was not equal-weighted and parallel, or the recognition of a 2-character word may involve serial processing from the first to the second character. As the repeat first and repeat second conditions did not differ in their N400 amplitude $(t(25)=1.0, P=0.35)$, this type of serial processing should not reflect semantic processing and was more likely to be orthographic in nature.

\section{General discussion}

The series of experiments reported here indicate that recognition of 2-character Chinese words elicited an early electrophysiological response N200. The response was widely distributed with a focus in central and parietal brain regions but not salient in occipital and temporal regions showing no clear sign of lateralization (Experiments 1 and 2). Repetition priming produced a very clear and large N200 enhancement effect (Experiment 3). Non-linguistic materials highly comparable in physical stimulus properties to the 2-character Chinese words did not elicit any similar response (Experiment 2), and the repetition of such items did not affect N200 either (Experiment 3). These results suggest that N200 shall reflect linguistic processing, as opposed to some low level sensori-perceptual processing. To further elucidate which linguistic dimensions among orthography, 

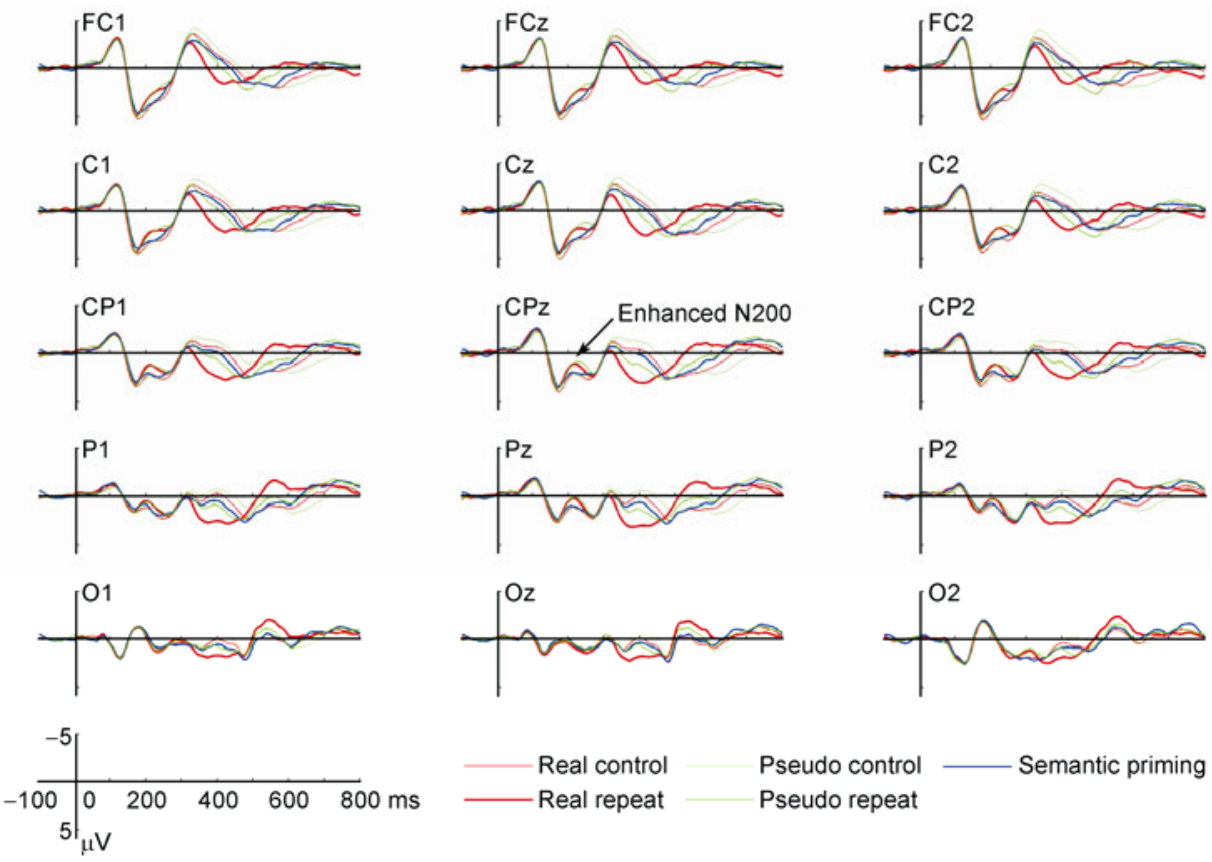

Pseudo control Pseudo repeat

Figure 5 Averaged ERP waveforms for all conditions in Experiment 5.
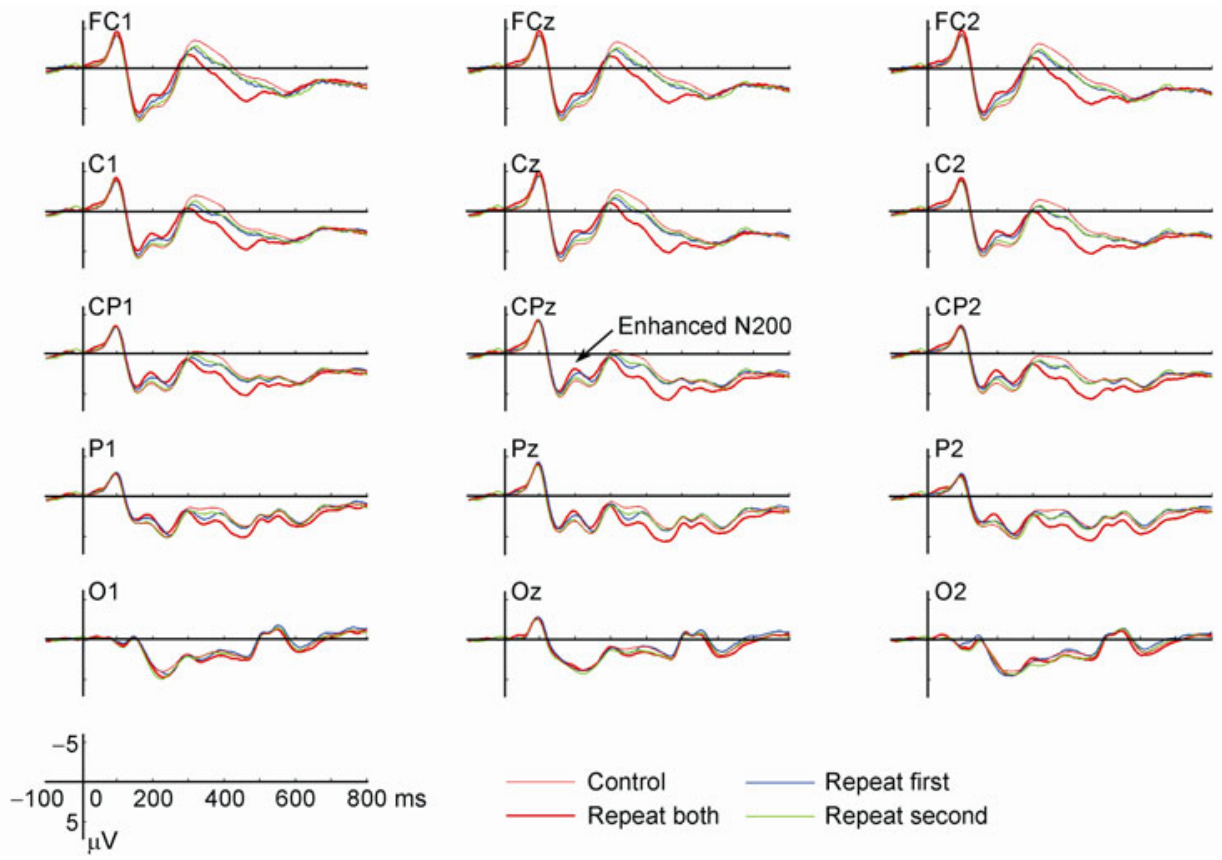

Figure 6 Averaged ERP waveforms for all conditions in Experiment 6.

phonology and semantics N200 was associated with, we showed in Experiments 4 and 5 that the N200 repetition enhancement effect was not affected by phonological or semantic priming, and cannot be attributed to the phonological or semantic relatedness between the prime and the target. Combined with results from Experiments 2 and 3, these results excluded the possibility that N200 was associated with phonology or semantics, indicating that it should reflect the remaining third dimension, i.e., orthographic pro- cessing. Experiment 6 provided more direct evidence for this conclusion by manipulating orthographic similarity between the prime and the target.

Using ERP, Huang et al. [34] recently studied the repetition effects of bi-syllabic Chinese words but did not report any similar negative deflection or negative repetition enhancement effect in the time window we observed N200 and its repetition enhancement. $\mathrm{Xu}$ [33] examined similar conditions involving repetition of bi-syllabic Chinese words 
auditorily presented and obtained similar results as Huang et al. What these findings mean is that even if the phonological representations associated with a 2-character word were directly activated via auditory presentation of its corresponding bi-syllabic speech form, no N200 or related N200 repetition enhancement could be observed. This indicates that the N200 enhancement observed when a visual word was repeated (Figure 7) is very unlikely to have originated from repeated activation of the word's phonological repre- sentation. These 2 studies therefore provide strong support for the notion that the N200 reported in this paper is modality-specific and reflects indeed orthographic processing. Briefly, this N200 response cannot be attributed to phonological or semantic factors or other characteristics of the Chinese words such as they are complex words consisting of 2 meaningful units.

The cognitive neuroscience research on complex words are just starting in recent years for alphabetic scripts. Due to
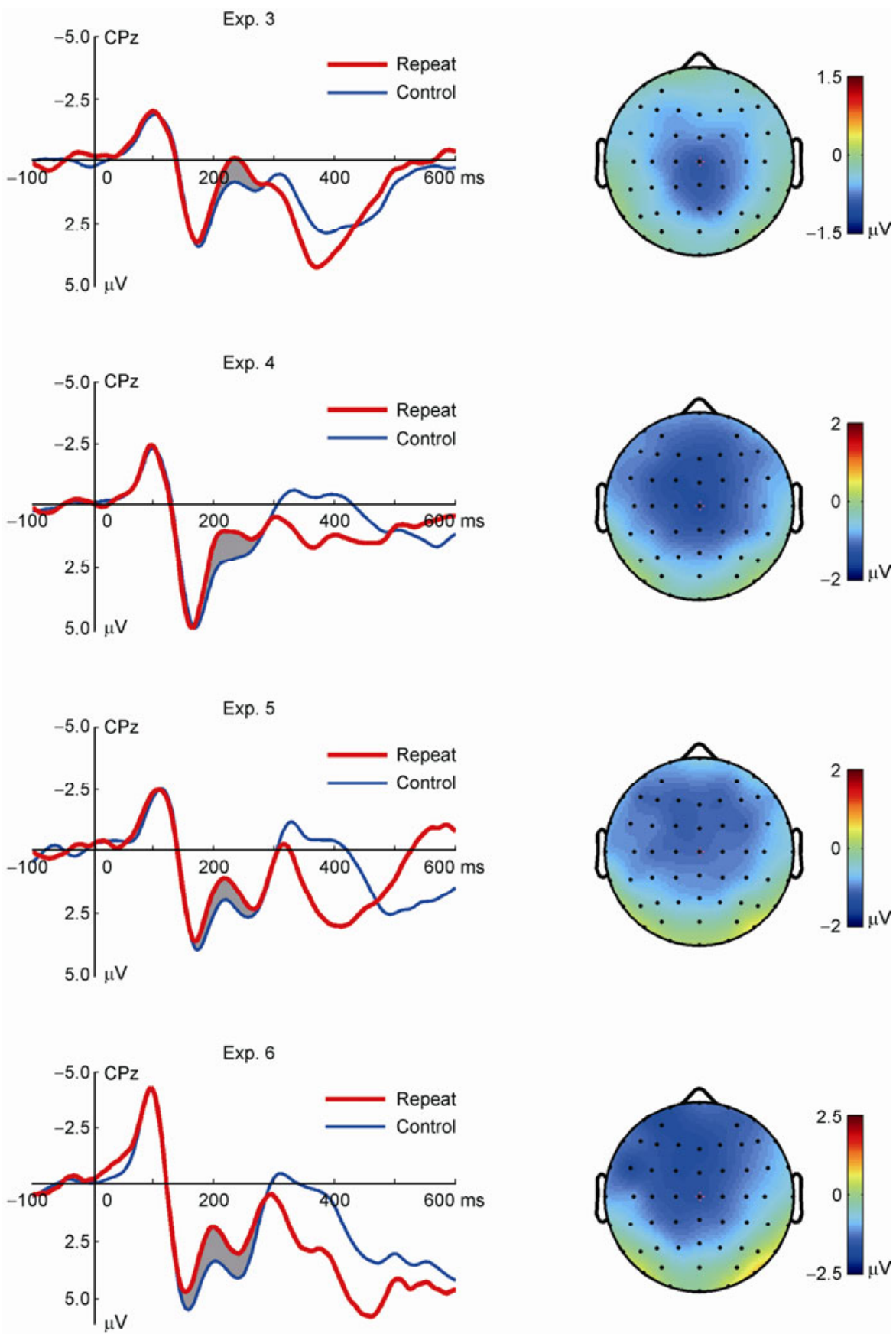

Figure 7 Averaged ERP waveforms in Experiments 3 to 6 for the real control and real repeat conditions in CPz and scalp topographies plotted based on the difference waves between these 2 conditions (mean amplitude difference in a $40 \mathrm{~ms}$ time window centered at the N200 peak). Cross indicates location of Cz. For Experiment 6, the real repeat condition means the repeat both condition. 
features of English as the dominant script examined (most complex words are inflectional or derivational), there has been even less work on compound words. So far we were able to identify only 2 ERP studies that seem related. Lehtonen et al. [35] compared early brain responses to inflected words and simple words in Finnish using a lexical decision task and did not observe any difference between the two. EI Yagoubi et al. [36] studied compound words in Italian and documented a $\mathrm{N} 2$ response in $\mathrm{Cz}$ with a rather late latency of about $300 \mathrm{~ms}$. This response was apparently different from the N200 reported here as it showed up for non-compound word as well.

In Experiments 1 and 4, we found that N200 amplitude was unaffected by the whole word frequency of a 2-character word, and was comparable across real words and pseudo-words constructed from 2 real characters, and that the N200 repetition effect was also similar across real and pseudo-words. Word frequency indicates familiarity with a stimulus. Pseudo-words as a whole unit can be regarded as a stimulus of zero frequency, or of very low familiarity. These results inform us that N200 is not sensitive to whether the orthography of a whole word is familiar or not. Further research is needed to find out exactly which aspects of orthographic processing N200 is sensitive to.

Review of the literature shows that extensive and systematic ERP research has been conducted on English and other alphabetic scripts in the past three decades, however, no study has reported effects similar to the present N200 and in particular its repetition enhancement effect [37-43]. A convincing case is that $\mathrm{Xu}$ et al. [33] followed exactly the same paradigm as a study on English and Spanish [44] except using Chinese words and Chinese participants. Figure 8 shows the clearly different results from these 2 studies involving an identical visual lexical decision task: while the Chinese words elicited a clear N200 response and a very salient repetition enhancement upon repetition, in the same time window, the English and Spanish words neither elicited any negative responses, nor showed any signs of negative enhancement at repetition.

For alphabetic scripts, the generally recognized ERP effects associated with repetition priming usually seem to emerge from $300 \mathrm{~ms}$ post-stimulus onset as a positive waveform shift. Prior to $300 \mathrm{~ms}$, there is usually no sign of any negative enhancement upon repetition. One line of research reports a N250 repetition effect [45], although it seems different from the N200 here as this N250 was usually found under the masked priming paradigm with a scalp distribution in temporal and occipital regions. More importantly, its repetition effect showed a positive shift. In English, there are also some reports of N200 considered to be associated with word form processing [46,47]. The basic paradigm used was to present prime-target word pairs with the prime sometimes masked. The results are not directly comparable to the present study as the SOA between the prime and the target was usually very brief (e.g., $200 \mathrm{~ms}$ ), making it a bit
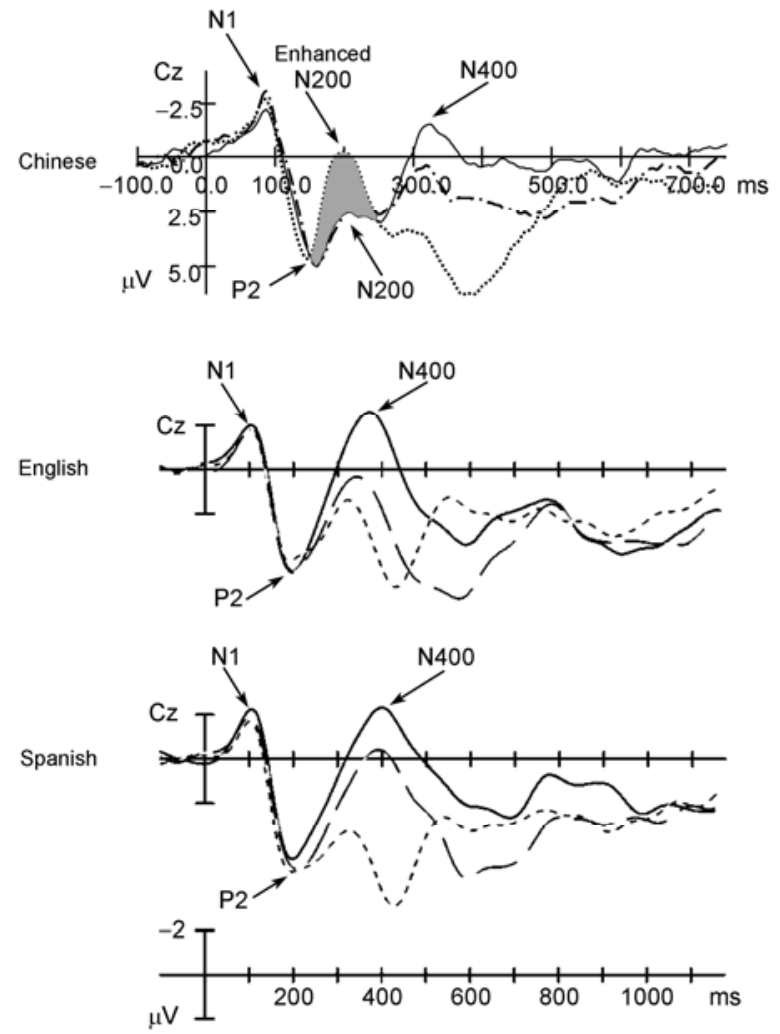

Figure 8 Results comparison of 2 studies adopting exactly the same visual lexical decision paradigm, one on Chinese and one on alphabetic scripts. The first presentation of 2-character Chinese words (solid line) elicited a clear N200 response and the repeated presentation (dotted line) elicited a salient N200 enhancement (the region marked with grey). In the same time window, the first presentation of English or Spanish words (solid line) did not elicit any negative deflection, and their second presentation (dotted line) did not elicit any negative enhancement either. With permission, the Chinese results were adapted from $\mathrm{Xu}$ [33], and the English and Spanish results from Alvarez et al. [44].

difficult to separate the ERP responses to the target from that to the prime. More importantly, the scalp distribution of these N200 responses was usually localized to occipital and temporal regions, very different from the widespread N200 response with a centro-parietal focus presented here.

Most ERP research on N2 is not about language materials but usually associated with non-linguistic factors such as perceptual mismatch, attention, control and inhibition [48,49]. Different from them, the N200 reported here is hard to be attributed to these factors as it was present in one script but not in others. Further, the latency of these N2 responses are usually about $300 \mathrm{~ms}$, much later than the N200 presented here. To identify an ERP component, other than its polarity, latency, and scalp distribution, one needs also to consider the kind of experimental manipulations that would affect the corresponding ERP response. Different negative deflections, although occurring in the similar 200-ms time window, may reflect very different mental processes. To avoid confusion, we tentatively refer the N200 reported here as the "centro-parietal N200" based on the characteristics of its scalp 
distribution to distinguish it from other negative waves in the same time window. With the accumulation of more relevant research data, this name may be adjusted to reflect a more thorough understanding of the component.

As the most important ERP finding in language research, the reduction of N400 at repetition priming is also one of the most significant ERP effects. We adopted the standard visual lexical decision paradigm that has been used extensively in the literature studying alphabetic scripts, and we only changed the materials to Chinese words and the participants to Chinese speakers. Other than replicating the classic N400 repetition effect, we repetitively recorded this early, large-sized, and widespread repetition effect going opposite to the N400 repetition effect. If similar effects were present in English or other alphabetic scripts, it would be hard to understand why so many existing studies failed to show it, even when some of them used highly comparable experimental paradigms as ours. In contrast, in the nonsystematic and very small ERP literature on Chinese word recognition, there were already several studies clearly documenting the N200 and its repetition enhancement effect. This tends to suggest that N200 may be an ERP response specific to Chinese word recognition and is simply not present for alphabetic scripts such as English.

The typical explanation of the priming effect is that prior exposure to a stimulus facilitates its later processing, and consequently repetition priming is often associated with reduction in brain responses and neural activation at the second presentation. Repetition of unfamiliar materials may occasionally produce neural enhancement $[50,51]$. The present research was different, however, in that all the materials used were highly familiar. Repeated presentation of a 2-character Chinese word produces N400 amplitude reduction and N200 amplitude enhancement. This is a very intriguing phenomenon and may offer a brand new perspective of the mechanisms of neural priming. In the ERP literature, there has been only one similar finding from Schweinberger et al. [52] who found a N250 repetition enhancement for face repetition, localized to a small area in the occipitotemporal regions and more lateralized to the right hemisphere. Schweinberger et al. suggested that the effect may reflect transient activations associated with face recognition but did not offer any explanation as to why it was an enhancement. What they emphasized was that the presence of this repetition effect indicates that recognition of individual face exemplars has been achieved in the N250 time window. The N270 response prior to this time window has been widely studied and known to be sensitive to face stimuli. However, N170 is not sensitive to face repetition. That is, for successive presentation of 2 faces, regardless of their being the same face or 2 different faces, the same N170 response would be elicited. In other words, N170 may only indicate that the visual system is able to tell whether or not a stimulus belongs to the face category, but has not got to the point to identify it as a specific face or an individual exem- plar of the face category. In comparison, that the N250 is sensitive to repetition indicates that the visual system is able to discriminate whether the 2 faces successively presented are the same or different, that is, already able to discriminate between different individual faces. Such discrimination should be based on the structural descriptions of the stimulus pattern, as opposed to its simple physical properties. This is because other ERP components that occur earlier than N170 or N250 and are known to code perceptual characteristics do not show repetition effects.

Based on the same logic, that the centro-parietal N200 is sensitive to word repetition indicates that as a neural response, it is able to differentiate between the Word A Word B (non-repeat control) pair and the Word A-Word A (repeat) pair, or in the around $200 \mathrm{~ms}$ time window, the visual system is able to discriminate between Word A and Word B to know that they are 2 different individual exemplars of the word category. As its repetition effect is not sensitive to phonological and semantic priming, N200 should reflect identification of a word's orthographic representation at the level of individual exemplar. This means that the recognition of the Chinese word's orthography has been achieved $200 \mathrm{~ms}$ after the word's presentation. Given the subsequent N400 is sensitive to word frequency, can discriminate real and pseudo-words, and reflects lexical level semantic access, the combination of the repetition effect of N200 and N400 locates the period between N200 and N400 to be the interval where Chinese word recognition progresses from orthographic recognition to semantic access, depicting a rather clear time course of the recognition process.

Other than being sensitive to faces, N170 sometimes showed strong responses to visual words $[53,54]$. In the present study, in occipital and temporal electrodes where N170 is typically recorded we also found clear N170 responses, which, however, were very different from N200 in not showing any repetition effect. In addition, the N170 elicited with word stimuli is usually locally distributed with a focus in posterior brain regions such as occipital and temporal regions, showing some level of left lateralization. Differently, the N200 presented here was very widespread with a focus in parietal and central regions. Therefore, it is unlikely that the centro-parietal N200 was a simple temporal delay of the N170 response. To summarize the above discussion, if N170 indeed indicates identification of the word stimuli at the categorical level, it may be combined with the centro-parietal N200, and N400 to mark the 3 major stages of Chinese word recognition.

It is reasonable to speculate that, around $200 \mathrm{~ms}$ poststimulus onset, both Chinese words and faces have achieved recognition of within-category individual exemplars, and that N200 or N250 reflects the process to construct the structural description necessary for stimulus identification. However, compared with the N250 effect for faces, the N200 repetition effect for Chinese words is of a much larger size and with a much broader distribution, extending be- 
yond early visual cortical regions to more advanced visual regions such as the parietal region, suggesting that the recognition of Chinese characters may be more complicated than face recognition, or at least with a rather different mechanism. In comparison, for non-face natural objects and words in alphabetic scripts such as English, no similar repetition enhancement effect has been observed, suggesting that their recognition may be with qualitatively different mechanisms from that for faces and Chinese words. Although both are linguistic materials, the visual processing involved in recognizing English and Chinese words may be rather different. For example, the orthographic processing of English words involves discriminating the one-dimensional linear combinations of several dozens of letters, and shall be associated with relatively simple structure coding. In comparison, orthographic processing in Chinese requires quantitatively the discrimination of about 5000 common characters, and structurally the extraction of 2-dimensional shape and spatial configuration information at the radical, single character, and multiple character levels, and seems to be more similar to what is involved in complex spatial and scene perception.

Recently, Zhang [55] proposed a meaning spelling theory of Chinese characters intended to provide a new conceptualization of written Chinese from a psychological point of view. The theory argues that at the vocabulary level, the Chinese script is a meaning spelling script that combines basic meaning-indicating units (meaning primitives) to construct new concepts. By this theory, the meaning spelling script and the alphabetic scripts constitute the two and the only 2 logical types of mature human written language and cannot be converted to each other without significant loss of communication efficiency. In particular, the alphabetic scripts are essentially an auditory language rooted in the auditory modality and constrained by intrinsic limits of the speech signals (one-dimensional linearity and a small number of basic phonological units). To construct an effective meaning spelling system, a written language must break away from the auditory modality and to capitalize on the visual modality with much more processing power, and also it must take advantage of the most powerful aspect of human vision, which is the ability in 2-dimensional pattern recognition. With a series of arguments, the meaning spelling theory predicts that, compared with alphabetic scripts, visual word recognition in Chinese should engage significantly more visual processing in its neural implementation.

Early orthographic processing shall be present for words in both Chinese and alphabetic scripts. However, the N200 associated with word form processing in alphabetic scripts was not sensitive to stimulus repetition. Based on the central logic adopted in the series of research of Schweinberger et al., such processing of word form may achieve category classification, i.e., able to classify a stimulus as a letter string, but not precise enough to discriminate different letter strings (words) to truly achieve recognition at the level individual word forms. In sharp contrast to this, the N200 elicited by the Chinese words was very sensitive to stimulus repetition, indicating the completion of individual word form recognition, that is to say, orthographic identification of a Chinese word should have completed $200 \mathrm{~ms}$ after the word was presented, seemingly earlier than that for words in alphabetic scripts. This may be because Chinese word recognition makes more use of the highly parallel visual modality with much more processing power, as opposed to the auditory modality emphasizing serial processing with relatively weaker power. Alternatively, earlier completion may suggest the processing of Chinese orthography is simpler, although this would contradict the scalp distribution of N200 in the 2 types of scripts. While N200 in English is confined to occipital and temporal regions, N200 in Chinese appears in parietal regions associated with complex visual analysis such as scene and spatial perception, suggesting that recognition of Chinese orthography may surpass the processing capability of occipital and temporal regions and require more processing resources and more advanced cortical regions.

Based on these considerations, we think that the discovery of N200 indicates that for Chinese word recognition, there exists an early stage of orthographic processing involving rather widespread and advanced visual processing, and this processing stage is simply not present for word recognition in alphabetic scripts based on letters or phonological units. This suggests that the former emphasizes more of visual processing than does the latter, which is consistent with a central prediction from the meaning spelling theory that written Chinese is a more thorough visual language compared with alphabetic writing systems. Based on our analysis of the literature, the centro-parietal N200 seems to be the clearest neural marker so far identified revealing distinctive processing mechanisms underlying word recognition across Chinese and alphabetic scripts. Its discovery reinforces the meaning spelling theory and the 2 combined argues both theoretically and empirically for the uniqueness of the Chinese language, and provides strong evidence that written Chinese and alphabetic scripts are inherently different.

As the meaning spelling theory can account for the presence of the centro-parietal N200, and discovery of the centro-parietal N200 also supports the meaning spelling theory, the 2 reinforce and strengthen each other. Still, they represent 2 independent research products, and one cannot be logically deduced from the other, so they may not be necessarily connected. For example, other theories may be developed to explain N200, and if Chinese is a visual language as the meaning spelling theory argues, it may manifest this characteristic in other ways of neural implementation. Presently, the meaning spelling theory offers one possible interpretation of the centro-parietal N200 phenomenon, to be confirmed or refuted in future research. 
This work was supported by the National Natural Science Foundation of China (30670702) and the Direct Grant from the Chinese University of Hong Kong (2020940).

1 Zhou Y G. The Developmental History of Scripts in the World (in Chinese). Shanghai: Shanghai Education Press, 1997

2 Wang W S Y. The Chinese language. Scient Am, 1973, 228: 50-60

3 Tzeng O, Hung D, Cotton B, et al. Visual lateralization effect in reading Chinese characters. Nature, 1979, 282: 499-501

4 Guo K J, Yang Q Z. "Both-hemisphere effect" in the cognition of Chinese characters (in Chinese). Acta Psychol Sin, 1995, 27: 78-83

5 Peng D L, Shu H, Chen H C. The Cognitive Studies on the Chinese Language (in Chinese). Jinan: Shandong Education Press, 1997

6 Peng D L. Cognitive Research on the Chinese Language: From Cognitive Science to Cognitive Neuroscience (in Chinese). Beijing: Beijing Normal University Press, 2006

7 Wu J T, Liu I M. Chinese lexical access. In: Bond M H, ed. The Handbook of Chinese Psychology. Hong Kong: Oxford University Press (China) Ltd, 1996. 30-42

8 Gazzaniga M S, Ivry R B, Mangun G R. Cognitive Neuroscience. Zhou X L, Gao D G, et al., translation. Beijing: China Light Industry Press, 2011

9 Yang Y M, Liang D D, Gu J X, et al. A neurolinguistic study on the classification of nouns and verbs in Chinese (in Chinese). Ling Sci, 2002, 1: 31-46

10 Wei J H, Kong P Z, Zhang D S, et al. The ERP characteristics of meaning association and the identification of known and unknown Chinese characters in the whole visual field (in Chinese). Acta Psychol Sin, 1995, 27: 413-419

11 Peng D L, Xu D, Jin Z, et al. Neural basis of the non-attentional processing of briefly presented words. Hum Brain Mapp, 2003, 18: 215-221

12 Lee C Y, Tsai J L, Kuo W J, et al. Neuronal correlates of consistency and frequency effects on Chinese character naming: An event-related fMRI study. NeuroImage, 2004, 23: 1235-1245

13 Zhang J X, Zhuang J, Ma L F, et al. Semantic processing of Chinese in left inferior prefrontal cortex studied with reversible words. NeuroImage, 2004, 23: 975-982

$14 \mathrm{Hu}$ W, Lee H L, Zhang Q, et al. Developmental dyslexia in Chinese and English populations: Dissociating the effect of dyslexia from language differences. Brain, 2010, 133: 1694-1706

15 Barber H, Kutas M. Interplay between computational models and cognitive electrophysiology in visual word recognition. Brain Res Rev, 2007, 53: 98-123

16 Zhang J J, Zhang H C, Peng D L. Meaning retrieval of Chinese characters during classification (2) (in Chinese). Acta Psychol Sin, 1991, 2: $139-144$

17 Song H, Zhang H C, Shu H. The developmental shift of the role of graphic code and phonetic code in Chinese reading (in Chinese). Acta Psychol Sin, 1995, 27: 139-144

18 Ding G S, Peng D L, Taft $\mathrm{M}$. The nature of the mental representation of radicals in Chinese: A priming study. J Exp Psychol Learn Memo Cogn, 2004, 30: 530-539

19 Ding G S, Peng D L. Mental recognition of Chinese words in reverse order: The relationship between whole word processing and morphemic processing (in Chinese). Contemp Linguist, 2006, 8: 36-45

20 Luck S. An Introduction to Event-related Potentials Technique. Fan S L, Ding Y L, Qu Z, et al., translation. Shanghai: East China Normal University Press, 2009

21 Liu Y N, Shu H. ERP and language (in Chinese). Adv Psychol Sci, 2003, 11: 296-302

22 Zhang J X, Kong L Y, Zhang Q, et al. An early ERP component for compound word processing. In: the 13th International Conference on the Processing of East Asian Languages (ICPEAL), Oct 9-11, 2009, Beijing

23 Zhang J X, Kong L Y, Zhang Q, et al. One novel ERP component brings three discoveries in language, memory, and attention. In: Annual Meeting of the Human Brain Mapping Society, June 6-11, 2010,
Barcelona

24 Modern Chinese Word Frequency Dictionary. Beijing: Beijing Language Institute Press, 1986

25 Kutas M, Federmeier K D. Thirty years and counting: Finding meaning in the N400 component of the event-related brain potential (ERP). Ann Rev Psychol, 2011, 62: 621-647

26 Zhang Q, Guo C Y, Ding J H, et al. Concreteness effects in the processing of Chinese words. Brain Lang, 2006, 96: 59-68

27 Zhang Q, Zhang J X, Kong L Y. An ERP study on the time course of phonological and semantic activation in Chinese word recognition. Int J Psychophysiol, 2009, 73: 235-245

28 Zhang Q, Ding J H, Guo C Y. ERP difference between processing of nouns and verbs (in Chinese). Acta Psychol Sin, 2003, 35: 753-760

29 Hsu C H, Tsai J L, Lee C Y, et al. Orthographic combinability and phonological consistency effects in reading Chinese phonograms: An event-related potential study. Brain Lang, 2009, 108: 56-66

30 Maeno T, Gjini K, Iramina K, et al. Event-related potential P2 derived from visual attention to the hemi-space, Source localization with LORETA. Int Cong Seri, 2004, 1270: 262-265

31 Schacter D L, Wig G S, Stevens W D. Reductions in cortical activity during priming. Curr Opin Neurobiol, 2007, 17: 171-176

32 Henson R N A, Rugg M D. Neural response suppression, hemodynamic repetition effects, and behavioural priming. Neuropsychologia, 2003, 41: 263-270

$33 \mathrm{Xu} \mathrm{G} \mathrm{F}$. Language Presentation and Language Switching Effects of Less Proficient Chinese-English Bilinguals: An ERPs Study (in Chinese). Master thesis. Tianjin: Tianjin Normal University, 2008

34 Huang X J, Zhang Q, Ding J H, et al. Effect of inter-item lag on spoken word recognition: An event-related potential study (in Chinese). Acta Psychol Sin, 2011, 43: 599-607

35 Lehtonen M, Cunillera T, Rodriguez-Fornells A, et al. Recognition of morphologically complex words in Finnish: Evidence from eventrelated potentials. Brain Res, 2007, 1148: 123-137

36 EI Yagoubi R, Chiarelli V, Mondini S. Neural correlates of Italian nominal compounds and potential impact of headedness effect: An ERP study. Cogn Neuropsychol, 2008, 25: 559-581

37 Karayanidis F, Andrews S, Ward P, et al. Effects of inter-item lag on word repetition: An event-related potential study. Psychophysiology, 1991, 28: 307-318

38 Doyle M C, Rugg M D, Wells T. A comparison of the electrophysiological effects of formal and repetition priming. Psychophysiology, 1996, 33: 132-147

39 Doyle M C, Rugg M D. Word repetition within- and across-visual fields: An event-related potential study. Neuropsychologia, 1998, 36: 1403-1415

40 Rugg M D, Nieto-Vegas M. Modality-specific effects of immediate word repetition: Electrophysiological evidence. Neuroreport, 1999, 10: 2661-2664

41 Strien J, Peter P, Verkoeijena N, et al. Electrophysiological correlates of word repetition spacing: ERP and induced band power old/new effects with massed and spaced repetitions. Int J Psychophysiol, 2007, 66: 205-214

42 Huber D E, Tian X, Curran T, et al. The dynamics of integration and separation: ERP, MEG, and neural network studies of immediate repetition effects. J Exp Psychol Hum Percept Perform, 2008, 34: 1389-1416

43 McDonald C R, Thesen T, Carlsonc C, et al. Multimodal imaging of repetition priming: Using fMRI, MEG, and intracranial EEG to reveal spatiotemporal profiles of word processing. NeuroImage, 2010, 53: 707-717

44 Alvarez R P, Holcomb R J, Grainger J. Accessing word meaning in two languages: An event-related brain potential study of beginning bilinguals. Brain Lang, 2003, 87: 290-304

45 Holcomb P J, Grainger J. On the time course of visual word recognition: An event-related potential investigation using masked repetition priming. J Cogn Neurosci, 2006, 18: 1631-1643

46 Grossi G, Coch D. Automatic word form processing in masked priming: An ERP study. Psychophysiology, 2005, 42: 343-355

47 Brown C M, Hagoort P, Chwilla D J. An event-related brain potential 
analysis of visual word priming effects. Brain Lang, 2000, 72: 158190

48 Patel S H, Azzam P N. Characterization of N200 and P300: Selected studies of the event-related potential. Int J Med Sci, 2005, 2: 147-154

49 Folstein J R, Petten C V. Influence of cognitive control and mismatch on the N2 component of the ERP: A review. Psychophysiology, 2008, 45: $152-170$

50 James T W, Gauthier I. Repetition-induced changes in BOLD response reflect accumulation of neural activity. Hum Brain Mapp, 2005, 27: 37-46

51 Grill-Spector K, Henson R, Martin A. Repetition and the brain: Neural models of stimulus-specific effects. Trends Cogn Sci, 2006, 10: 14-23

52 Schweinberger S R, Huddy V, Burton A M. N250r: A face-selective brain response to stimulus repetitions. Neuroreport, 2004, 15: 15011505

53 Bentin S, Mouchetant-Rostaing Y, Giard M H, et al. ERP manifestations of processing printed words at different psycholinguistic levels: Time course and scalp distribution. J Cogn Neurosci, 1999, 11: 235260

54 Cao X H, Li S, Zhao J, et al. Left-lateralized early neurophysiological response for Chinese characters in young primary school children. Neurosci Lett, 2011, 492: 165-169

55 Zhang J X. The meaning spelling theory of Chinese characters: Insight into the nature of written Chinese from the perspective of cognitive psychology (in Chinese). J South China Normal University (Social Science Edition), 2011, 4: 5-13

Open Access This article is distributed under the terms of the Creative Commons Attribution License which permits any use, distribution, and reproduction in any medium, provided the original author(s) and source are credited. 